\title{
Bilateral Adrenal Mass Mimicking Adrenocortical Carcinoma
}

\author{
Sule CANLAR 1 , Celal IDEMEN ${ }^{2}$, Aysun YALCI ${ }^{3}$, Alpay AZAP ${ }^{3}, \underline{\text { Murat Faik ERDOGAN }}$ \\ -1 Department of Endocrinology and Metabolic Diseases, Ankara University of Medicine \\ -2 Department of Internal Medicine, Ankara University of Medicine \\ -3 Clinical Microbiology and Infectious Diseases, Ankara University of Medicine
}

\section{Objectives}

Bilateral adrenal mass presents a unique diagnostic challenge and carries additional risk of being metastasis. The differential diagnosis of bilateral adrenal mass include primary or secondary neoplastic disorders (adrenocortical carcinoma, lymphoma, metastases), pheochromocytoma, longstanding congenital adrenal hyperplasia and macronodular adrenal hyperplasia, infections such as tuberculosis, histoplasmosis and blastomycosis. Here we report a man presenting with bilateral adrenal mass and adrenal failure.

\section{Case \\ Presentation}

35 year-old male presented with weight loss, fever, emesis and headache. Laboratory findings showed hyperkalemia, hyponatremia, low cortisol serum levels and significantly elevated ACTH, CRP serum levels and ESR. Glucocorticoid treatment was initiated immediately. For evaluation of etiology of severe weight loss, CT disclosed bilateral adrenal mass, milimetric pulmonary nodules and cerebral-cerebellar nodulary lesions. Upon investigation, the patient complained testicular pain and swelling, and physical examination illustrated testicular mass ultrasound revealed epididimal thickening, heterogeneity and ductal hypoechoic nodulary lesions. For differential diagnosis, PET CT scan was performed and high adrenal mass uptakes (SUVmax:18,4) compatible with malignancies was detected. In the search for a definitive diagnosis, between metastatic adrenal carcinoma, metastatic carcinoma of unknown origin (lung, malignant melanoma etc) and infectious causes, ultrasound guided fine needle aspiration biopsy of the adrenal mass was carried out. Histopathological examination demonstrated necrotizing granulomatous inflammation consistent with tuberculosis. Anti-tuberculosis treatment was started as soon as possible and the patient had a remarkable recovery through several months.

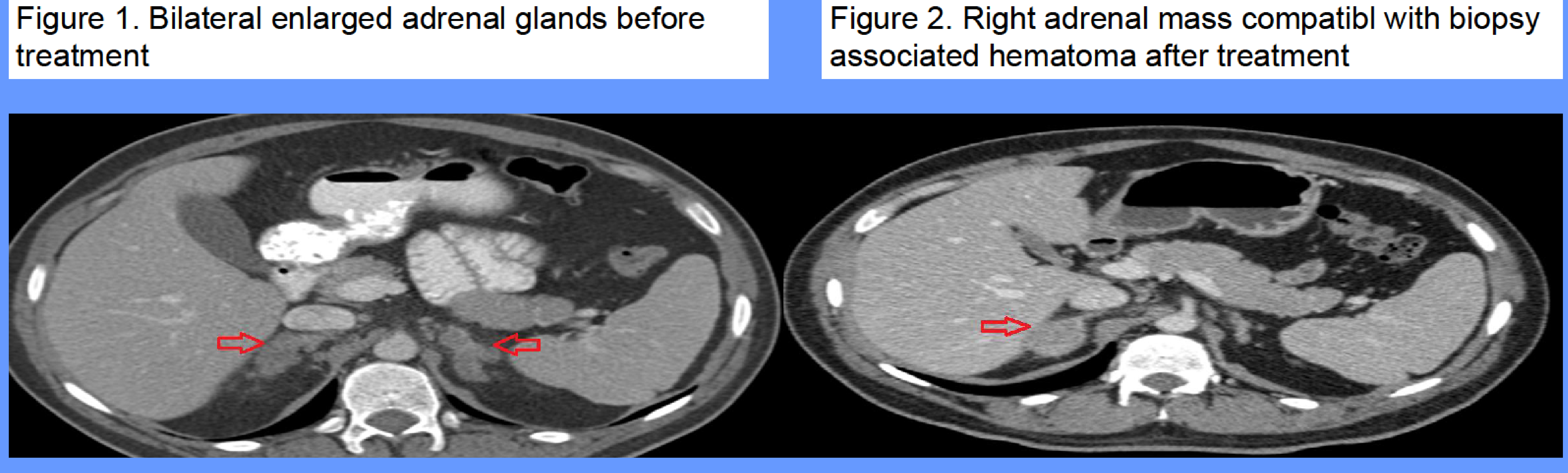

Conclusions

The most common causes of Addison's disease are idiopathic adrenal atrophy and adrenal tuberculosis. Globally, tuberculosis prevalence decreases and it is reported that TB prevalence in 2015 was $42 \%$ lower than it used to be in 1990. However, tuberculosis is still an important health problem. Adrenal tuberculosis should be taken into consideration when adrenal failure and bilateral adrenal involvement is detected

1. P. Gupta, A. Bhalla, R. Sharma, Bilateral adrenal lesions, Journal of Medical Imaging and Radiation Oncology Volume 56, Issue 6December 2012 Pages 636-645

2. Jagriti Upadhyay, Praveen Sudhindra, George Abraham, and Nitin Trivedi, Tuberculosis of the Adrenal Gland: A Case Report and Review of the Literature of Infections of the Adrenal Gland International Journal of Endocrinology

Volume 2014 (2014), Article ID 876037

3. WHO 2015 Global Tuberculosis Report 